\title{
Citrate: How to get started and what, when, and how to monitor?
}

\author{
Patrick M. Honore ${ }^{1}$, David De Bels ${ }^{1}$, Thierry Preseau ${ }^{2}$, \\ Sebastien Redant ${ }^{1}$, Herbert D. Spapen ${ }^{3}$ \\ IICU Department, Centre Hospitalier Universitaire Brugmann, Brussels, Belgium; \\ ${ }^{2}$ Emergency Dept, Centre Hospitalier Universitaire Brugmann, Brussels, Belgium; \\ ${ }^{3}$ Universitair Ziekenhuis Brussel, VUB University, Brussels, Belgium
}

Address for Correspondence: Prof. Patrick M. Honore, MD, PhD, FCCM, Professor of Medicine \& Co-Director, ICU Department, Centre Hospitalier Universitaire Brugmann, Place Van Gehuchtenplein, 4, 1020, Brussels, Belgium.

E-mail: Patrick.Honore@CHU-

Brugmann.be

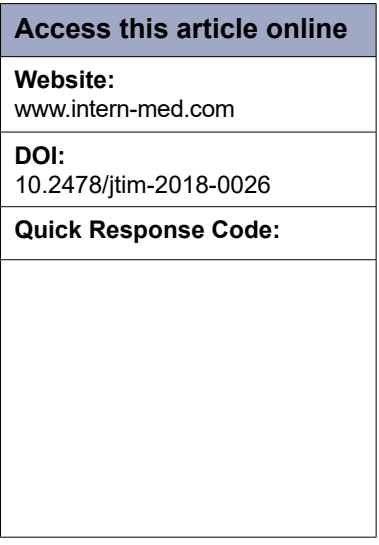

\section{ABSTRACT}

In most of the case, regional citrate anticoagulation is using diluted citrate around $1 \%$ depending on the types used in clinical practice. Diluted citrate is much more safer when compared to highly concentrated citrate around $4 \%$ or even more. In clinical practice, trisodium citrate is used in high concentration (around 30\%) as a bactericidal agent with anticoagulant properties for locking deep venous catheters used in hemodialysis (HD; close to $25-30 \%$ of citrate). In this review article, buffer and anticoagulant potential of citrate are discussed during renal replacement therapy in critically ill patients with particular focus on the practical approach at the bedside.

Key words: regional citrate anticoagulation, diluted citrate, monitoring, metabolic complications

\section{UTILIZATION OF CITRATE: ANTICOAGULANT, BUFFER AND BACTERICIDAL AGENT}

Citrate is the conjugate base of citric acid. It is an intermediate conjugate in the citric acid cycle (Krebs cycle or tricarboxylic acid cycle). After the pyruvate dehydrogenase complex forms acteyl-coenzyme A (acetyl-CoA), citrate synthase catalyzes the condensation of oxaloacetate with acetyl-CoA to form citrate. Citrate continues in the cycle with eventual regeneration of oxaloacetate, which can combine with another molecule of acetyl-CoA and continue cycling. In clinical practice, trisodium citrate is used in high concentration (around 30\%) as a bactericidal agent with anticoagulant properties for locking deep venous catheters used in hemodialysis (HD; close to 25-30\% of citrate). ${ }^{[1-2]}$

In this article, buffer and anticoagulant potential of citrate are discussed during renal replacement therapy in critically ill patients with particular focus on the practical approach at the bedside.

\section{HOW DOES CITRATE WORK UNDER CLINICAL CONDITIONS?}

To avoid early clotting and particularly clogging of both the filter and the extracorporeal circuit during continuous renal replacement therapy (CRRT), longterm anticoagulation is often required. Until recently, unfractionated or lowmolecular-weight heparins were generally used. However, prolonged systemic anticoagulation may be hazardous or even contraindicated in a substantial number of critically ill patients because of recent surgery or trauma, active or recent mucosal bleeding, severe coagulopathy or heparin-induced thrombocytopenia, and thrombosis. In these patients, anticoagulation of the CRRT circuit can be obtained by using citrate as a regional or even as a local anticoagulant (the so-called citrazet membrane). Citrate-administered pre-filter prevents coagulation by chelating ionized calcium (and also magnesium) and may induce deep hypocalcemia into the filter. Part of the calcium-citrate complex is lost in the ultrafiltrate or in the effluent of the dialysate; although remaining amounts 
enter the systemic circulation, they are diluted in the venous blood and become available for metabolization, mainly in the liver. Actually, the part available for metabolization is really minimized. Ionized calcium levels rise in the systemic circulation because of the dilution of extracorporeal blood returning to patients' circulation, the replacement of calcium, and the liberation of calcium from calciumcitrate and magnesium-citrate complexes when citrate is metabolized. As a result, a systemic effect on coagulation does not occur unless specific conditions are present, such as impaired liver or skeletal muscle function (e.g., after a cardiac arrest). ${ }^{[1-2]}$ Citrate is converted to citric acid in the liver, yielding three molecules of bicarbonate (although this may considerably vary depending on conditions other than liver failure) and is either metabolized in the citric acid (Krebs or tricarboxylic acid) cycle in liver, skeletal muscle $(20 \%)$, and renal cortex $(5 \%)$ or metabolized to glucose (gluconeogenesis). ${ }^{[1-2]}$ On top of this, citrate can also inhibit platelet function, which enhances its anticoagulant potential. Although this effect cannot be monitored (regarding platelet inhibition), this can also be a problem in some (very few) parts of the circuit. Anticoagulation with citrate has complex metabolic consequences. Apart from its use as an anticoagulant, citrate also acts as a buffer and is, nowadays, increasingly used as such. Potential drawbacks of trisodium citrate use are the substantial sodium load and the loss of calcium in the ultrafiltrate because of citrate binding, which is more prominent than during hemofiltration or HD with heparin. Furthermore, the amount of citrate lost during ultrafiltration varies with UF flow. Consequently, the amount of buffer entering the systemic circulation varies accordingly. The amount of citrate varies also with blood flow, as significantly more citrate may be eliminated at higher blood flow. Under special circumstances, in particular if liver and skeletal muscles fail to metabolize citric acid, bicarbonate is not produced and citrate may accumulate. This actually occurs at a very low rate because extremely low concentration of citrate are used. ${ }^{[3]}$ In the light of the above, the composition of the replacement fluid has to be adjusted during citrate hemofiltration, metabolic monitoring is indispensable (every $4 \mathrm{~h}$ from start of treatment), and, given the lack of systemic anticoagulation, standard thrombosis prophylaxis must be provided unless contraindicated. Until recently, citrate was only administered separately as a concentrated solution before the predilution line, thereby increasing the risk of severe accumulation in patients with impaired hepatic function. Lately, a trend is observed toward the use of manufactured industrial solutions with low citrate concentrations given together with the predilution. ${ }^{[4]}$ This dramatically reduces the risk of subsequent side effects and paves the way for more generalized use of citrate as an anticoagulant.
The development of computerized systems adapting dosing of citrate to blood flow, pre- and postdilution flow, and ultrafiltration or dialysate flow will facilitate a more flexible clinical use without additional risks. The introduction of these computerized systems to a wider variety of devices (working nowadays with citrate) will undoubtedly improve patient safety without being more labor intensive for the nursing staff. Until now, the low citrate dose in predilution mode provided efficient anticoagulation but poorly controlled metabolic acidosis. Our major aim is to dissociate the effect of citrate as an anticoagulant from its role in generating bicarbonate, that is, to give sufficient citrate through the predilution mode to completely correct acidosis while avoiding citrate-related complications. ${ }^{[5]}$

\section{INDICATIONS AND CONTRAINDICATIONS FOR CITRATE AS AN ANTITHROMBOTIC AGENT DURING CRRT IN ICUS}

It is a well-established practice in intensive care units (ICUs) nowadays to refrain from using any form of heparin in patients with active or recent bleeding. The high-risk group of patients at risk is not only consisting of patients following recent surgery or trauma but do also consist of a lot of medical conditions leading to high risk of bleeding such as hemorrhagic diathesis, severe mucous ulcerations, intracranial spontaneous bleeding, uremic pericarditis, severe (diabetic) retinopathy, malignant hypertension, uncontrollable coagulopathy, and other hematological diseases. The same measures are invoked in case of heparininduced thrombocytopenia and thrombosis (HIT) though, as HIT can be associated with a pro-coagulant state, some form of alternate systemic anticoagulation is necessary. Danaparoid or a first, second, and even third generation of thrombin inhibitors such as argatroban are an option. However, controversy remains with regard to potential cross-reactivity between heparin and both danaparoids and thrombin inhibitors. Here too, low-dose citrate could conceivably play a role in systemic anticoagulation. Contraindications for citrate use relate to diminished citrate clearance, which is observed either in severe liver failure or in highly reduced muscle perfusion, for example, after prolonged cardiac arrest. Under close monitoring, a lower citrate dose (e.g., starting with $50 \%$ of the required dose and tapering according to ionized calcium levels in the circuit) could still be used in these conditions. ${ }^{[3]}$

A recent study in critically ill patients with liver cirrhosis demonstrated that citrate clearance, measured after a 2-h infusion of citrate of $500 \mathrm{mmol} / \mathrm{kg} / \mathrm{h}$ under adequate calcium chloride replacement, was roughly $50 \%$ lower 
than that in non-cirrhotic critically ill patients. ${ }^{[3]}$ It stands to reason that a reduced dose of citrate subject to more frequent monitoring of citrate accumulation can play a role in the cirrhotic patient as well as post-cardiac arrest. In the recent years, growing clinical experience and evidence has shown that patients with compensated liver cirrhosis could well tolerate anticoagulation with citrate. These patients are also at risk for bleeding when heparins or similar products are applied. Patients in septic shock with lactic acidosis, in whom limited metabolism of citrate can be foreseen because of disturbed liver and muscle perfusion, can generally be filtrated with citrate without signs of accumulation, on the condition that strict monitoring is provided and dosing errors are avoided. Moreover, the currently used much lower concentrations of citrate (18 $\mathrm{mmol} / \mathrm{L}$ ) represents only $10 \%$ of a $500-\mathrm{mmol} / \mathrm{kg} / \mathrm{h}$ dose. Under these conditions, the clearance of citrate in patients with liver cirrhosis may thus be only $25 \%$ of the "normal citrate clearance," although further proof is awaited.

Several systems for citrate anticoagulation in continuous venovenous hemofiltration $(\mathrm{CVVH})$ and continuous venovenous hemodiafiltration (CVVHDF) have been described. The two major options for substitution in CVVH are postdilution and predilution. Citrate is always administered prefilter because it is applied for anticoagulation. In the postdilution mode, citrate is infused as a trisodium citrate solution before the filter, while the replacement fluid contains low sodium and either no or a low buffer concentration as a bicarbonate infusion of
20-25 mmol/L. If necessary, additional calcium is infused via a separate line post-filter in order to avoid the risk of thrombosis inside the access catheter. In contrast, in predilution mode, citrate is added to the replacement fluid that contains no other buffer or a combination of citrate and bicarbonate, or infused as a separate trisodium citrate solution in addition to an adjusted replacement fluid with low bicarbonate solutions around $20 \mathrm{mmol} / \mathrm{L}^{.1-3]}$

Adding citrate to predilution fluid is advantageous, as it allows to lower concentration with the same dosage. In this mode, calcium has to be administered separately. No single randomized study comparing these different modes has yet been published. Therefore, any clinical decision regarding the choice between pre- and postdilution with citrate either added to the substitution fluid or infused over a separate line is not evidence based.

"Pros and cons" referring to both post- and predilution systems for CVVH are discussed throughout this section. Predilution reduces efficacy of clearance, which may be compensated by a higher filtrate flow. As shown in Table 1, the predilution system as described by Pallson (with citrate in the restitution fluid) is striking in its simplicity, whereas for CVVHDF, the citrate is infused separately as a trisodium citrate solution before the filter. Generally, a calcium-free dialysate is used and calcium is infused via a separate line, but Mitchell (Table 2) uses a calcium-containing dialysate. In some options, the replacement is administered in predilution as a citrate infusion.

\section{Table 1: Protocols of citrate anticoagulation in CVVH}

\begin{tabular}{|c|c|c|c|c|}
\hline Types & $\begin{array}{l}\text { Mear } \\
(\mathrm{mm}\end{array}$ & Blood flow (mL/min) & Composition o & tion fluid (mmol/L) \\
\hline $\begin{array}{l}\text { CVVH } \\
\text { Predilution } \\
\text { (Palsson, 1999) }\end{array}$ & 18.6 & 180 & $\begin{array}{l}\text { Citrate: } 13.3 \\
\mathrm{Na}: 140 \\
\mathrm{Cl}: 101.5 \\
\text { Mg: } 0.75 \\
\text { Glucose: } 1.1\end{array}$ & \\
\hline $\begin{array}{l}\text { CVVH } \\
\text { Postdilution } \\
\text { (Spijker, 1999) }\end{array}$ & 26.5 & 180 & $\begin{array}{l}\text { Na: } 100 \\
\mathrm{Cl}: 110 \\
\mathrm{Mg}: 0.8 \\
\mathrm{~K}: 4 \\
\mathrm{Ca}: 2 \\
\text { Glucose: } 4.4\end{array}$ & \\
\hline $\begin{array}{l}\text { CVVH } \\
\text { Postdilution } \\
\text { (Oudemans, 2002) }\end{array}$ & 35 & 200 & $\begin{array}{l}\mathrm{Na}: 110 \\
\mathrm{Cl}: 116 \\
\mathrm{Mg}: 0.52 \\
\mathrm{~K}: 2 \\
\mathrm{Ca}: 1.91 \\
\text { Bicarbonate: } 0 \\
\text { Lactate: } 3 \\
\text { Glucose: } 5.8\end{array}$ & $\begin{array}{l}\text { Na: } 140 \\
\text { Cl: } 111 \\
\text { Mg: } 0.75 \\
\text { K: } 2 \\
\text { Ca: } 1.25 \\
\text { Bicarbonate: } 35\end{array}$ \\
\hline $\begin{array}{l}\text { CVVH } \\
\text { Pre- and Postdilution } \\
\text { (Monchi, 2004) }\end{array}$ & 38 & 150 & $\begin{array}{l}\mathrm{Na}: 120 \\
\mathrm{Cl}: 122 \\
\mathrm{Mg}: 0.50 \\
\mathrm{~K}: 1\end{array}$ & \\
\hline
\end{tabular}




\begin{tabular}{|c|c|c|c|c|c|}
\hline Types & $\begin{array}{l}\text { Mean initial infusion of } \\
\text { citrate }(\mathrm{mmol} / \mathrm{h})\end{array}$ & Blood flow (mL/min) & $\begin{array}{l}\text { Composition of replacement } \\
\text { fluid ( } \mathrm{mmol} / \mathrm{L} \text { ) }\end{array}$ & \multicolumn{2}{|c|}{$\begin{array}{l}\text { Composition of dialysate } \\
\text { (mmol/L) }\end{array}$} \\
\hline $\begin{array}{l}\text { CAVHD } \\
\text { (Mehta, 1990) }\end{array}$ & 23.8 & $50-125$ & $\begin{array}{l}\mathrm{Na}: 154 \\
\mathrm{Cl}: 154\end{array}$ & \multicolumn{2}{|l|}{$\begin{array}{l}\mathrm{Na}: 117 \\
\mathrm{Cl}: 122.5 \\
\mathrm{Mg}: 0.75 \\
\mathrm{~K}: 4 \\
\text { Dextrose: } 2.5 \%\end{array}$} \\
\hline $\begin{array}{l}\text { CVVHD } \\
\text { (Tolwani, 2001) }\end{array}$ & 17.5 & $125-150$ & None & \multicolumn{2}{|l|}{$\begin{array}{l}\mathrm{Na}: 154 \\
\mathrm{Cl}: 154 \\
\mathrm{Mg}: 1 \\
\mathrm{~K}: 3\end{array}$} \\
\hline $\begin{array}{l}\text { CVVHD } \\
\text { (Meier, 2001) }\end{array}$ & 31.6 & $150-200$ & None & \multicolumn{2}{|l|}{$\begin{array}{l}\mathrm{Na}: 110 \\
\mathrm{Cl}: 110 \\
\mathrm{Mg}: 1 \\
\mathrm{~K}: 2\end{array}$} \\
\hline $\begin{array}{l}\text { CVVHD } \\
\text { (Bunchman, 2002) }\end{array}$ & 17 & 100 & None & \multicolumn{2}{|l|}{$\begin{array}{l}\text { Na: } 140 \\
\mathrm{Cl}: 106.5 \\
\text { Mg: } 0.75 \\
\text { Bicarbonate: } 35\end{array}$} \\
\hline $\begin{array}{l}\text { CVVHD } \\
\text { (Mitchell, 2003) }\end{array}$ & 30 & 75 & None & $\begin{array}{l}\text { Na: } 121 \\
\text { Cl: } 110 \\
\text { Mg: } 0.50 \\
\text { K: } 1.9 \\
\text { Ca: } 1.75 \\
\text { Bicarbonate: } 13 \\
\text { Lactate: } 2.9\end{array}$ & $\begin{array}{l}\text { Na: } 140 \\
\text { Cl: } 113 \\
\text { Mg: } 0.51 \\
\text { K: } 3.9 \\
\text { Ca: } 1.78 \\
\text { Bicarbonate: } \\
34 \\
\text { Lactate: } 2.96\end{array}$ \\
\hline
\end{tabular}

\section{HOW TO PRESCRIBE CITRATE AT THE BEDSIDE?}

A target citrate concentration of 3-6 mmol/L should be attained before the filter to reach an ionized calcium concentration below $0.35 \mathrm{mmol} / \mathrm{L}$ (or between 0.8 and $1.3 \mathrm{mg} / \mathrm{dL}),{ }^{[1-2]}$ which is imperative to avoid coagulation inside the filter and the circuit. As previously indicated, optimal prescription of citrate in any new protocol requires dose adjustment, among others, to blood flow. In the different systems described, citrate is either infused as a separate solution or included in the replacement fluid. In the latter and currently more popular option, as described for predilution, a fixed relation between citrate flow and blood flow is not guaranteed as the flow of the replacement fluid varies with the UF flow and the desired fluid removal. For instance, when UF flow diminishes or when a more negative fluid balance is desired, less citrate is administered and early clotting of the filter is precipitated. This might be detrimental for filter survival time. Theoretically, control of anticoagulation is optimal with citrate administered in a separate solution. In clinical practice, however, citrate diluted into the predilution fluid seems to be the most efficient and surely the safest option.

Several formulations of citrate solutions are currently available in the market, with trisodium citrate concentrations varying from $0.5 \%$ to $30 \%$. The lower the concentration is, the higher the volume infused (and to be stored in the department) but the lower is the risk of side effects due to citrate anticoagulation. Some solutions are commercially available, albeit not in all countries, which explains why they are often prepared by local hospital pharmacies or even at the bedside. Solutions also vary in the proportion of trisodium citrate and citric acid. A citrate dextrose-A (ACDA) solution is sometimes used, containing approximately $74.8 \mathrm{mmol} / \mathrm{L}$ of citrate, 38.1 $\mathrm{mmol} / \mathrm{L}$ of citric acid, $221 \mathrm{mmol} / \mathrm{L}$ of sodium, and $124 \mathrm{mmol} / \mathrm{L}$ of dextrose. As a comparison, recent $0.5 \%$ solutions do contain only $18 \mathrm{mmol} / \mathrm{L}$ of citrate. Therefore, an equimolar infusion of citrate may differ in amount of sodium infused and buffer potential of the solution. For citrate dosing, a fixed blood flow/citrate flow relation is often preferred and thus obviating the need for routine monitoring of anticoagulation in the extracorporeal circuit. If blood flow is kept constant and citrate dose is adjusted to blood flow, routine measurement is recommended only when initiating the technique in a department. After some clinical experience has been gained, monitoring circuit anticoagulation can be limited to special cases such as unexplained early filter clotting. Nevertheless, some centers prefer to measure anticoagulation in the extracorporeal circuit and routinely titrate citrate dose. Although still unproven, this may tend to increase filter lifespan. Extracorporeal anticoagulation can be monitored by measuring post-filter ionized calcium concentration (targeting below $0.35 \mathrm{mmol} / \mathrm{L}$ or between 0.8 and 1.3 $\mathrm{mg} / \mathrm{dL}$ ), activated clotting time (ACT), or activated 
partial thromboplastin time (aPTT). Several algorithms for adjusting the citrate infusion rate are described in the literature. Flexibility in using protocols tailored to specific patient needs definitely will improve with increasing clinical experience. Yet, continuous monitoring, even at extended intervals, of ionized calcium in the circuit is still accepted as the gold standard. On top of this, we still do not have available monitoring for platelet's citrate effect (outside the effect of citrate on classical coagulation), and indeed, this can affect a lot of "early venous gas trap chamber" with platelet thrombosis mainly. ${ }^{[1-4]}$

\section{DOSE EFFICACY OF CITRATE IN RELATION TO ACID-BASE BALANCE}

Citrate, in its currently used trisodium citrate form, acts as a buffer, as it is converted to citric acid, thereby generating sodium bicarbonate as follows:

In theory, $3 \mathrm{Na}$ citrate $+3 \mathrm{H}_{2} \mathrm{CO}_{3} \leftrightarrow$ Citric acid +3 $\mathrm{NaHCO}_{3}$, but in practice, this will depend on the type of solution used and, in fact, on the proportion of sodium and hydrogen in a given citrate solution. ${ }^{[1-4]}$

Citric acid (with a very short plasma half-life of around $5 \mathrm{~min}$ ) is then metabolized to $\mathrm{CO}_{2}$ and $\mathrm{H}_{2} \mathrm{O}$ by liver, kidney, and muscle cells. The formula shows that the net amount of the produced bicarbonate is dependent on the proportion of sodium and hydrogen in the citrate solution. The amount of citrate entering the systemic circulation depends on the net balance of the proportion of citrate infused and its loss in ultrafiltrate and dialysate. Clearance of citrate by ultrafiltration is equal to clearance of urea and, at least in some proportions, does not change when CVVH is switched to CVVHD (continuous venovenous hemodialysis). Subsequently, cellular uptake and metabolic degradation of citric acid will determine the acid-base balance. Metabolic acidosis inevitably ensues from citric acid accumulation and-according to the Stewart approachless strong anion accumulation will increase the strong ion difference $[(\mathrm{Na}+\mathrm{K}+\mathrm{Ca}+\mathrm{Mg})$ minus $(\mathrm{Cl}+$ lactate + other strong anions)]. In fact, composition of the citrate solution, citrate infusion rate, and loss of citrate (bound to calcium or magnesium) by filtration and further metabolism of citric acid will all influence acid-base balance during citrate CVVH. In postdilution with citrate infusion adjusted to a given blood flow and ideally kept constant during treatment, the amount of citrate, sodium, and bicarbonate lost through ultrafiltration depends on UF flow. If a higher UF rate is applied, more ions are filtered and less ions enter the systemic circulation. Conversely, if UF flow declines during the course of a single hemofiltration run, less ions are filtered and more ions enter the systemic circulation. The loss of buffer (anions) and cations in ultrafiltrate must be balanced by their content in the replacement fluid. In clinical practice, however, replacement fluid is adjusted to UF flow and measured acidbase state. In this way, the system is highly flexible and close metabolic control can be obtained. It is important to note that blood flow has an independent effect, in particular on the clearance of sodium and citrate. Importantly, a change in blood flow at a constant citrate flow also influences citrate clearance, because the concentration of citrate in the filter changes. This effect is not clinically relevant but may become more important when low concentrations of citrate (i.e., $0.5 \%$ citrate $/ 18 \mathrm{mmol} / \mathrm{L}$ ) are used. However, it is imperative to calculate solute balances for each combination of blood, citrate, and ultrafiltration flow and to adhere carefully to written protocols before implementing the method in the ICU. Anyway, close monitoring of ionized calcium should already allow sufficient control under those circumstances. In the predilution CVVH system of Pallson and Thoenen (Table 1) ${ }^{[5]}$ citrate is added to the replacement fluid and calcium is infused by a separate line. Citrate infusion is matched by the loss of buffer in the ultrafiltrate unless a larger amount of fluid is withdrawn. Although striking by its simplicity, a major drawback of the system is that the buffer cannot be titrated separately. This might be detrimental when buffer needs are increased (e.g., severe acidosis) or with increased loss of citrate and bicarbonate in ultrafiltrate (e.g., at higher ultrafiltration rates). In analogy to the model described by Pallson, ${ }^{[5]}$ Aspner proposed a replacement solution for predilution CVVH containing both citrate (15 $\mathrm{mmol} / \mathrm{L})$ and bicarbonate $(10 \mathrm{mmol} / \mathrm{L})$ for use in patients with severe acidosis due to shock. ${ }^{[3]}$ More details regarding the circuit were not described at the time. In practice, a citrate concentration of $15 \mathrm{mmol} / \mathrm{L}$ should be sufficient to account for the acidosis observed in an ICU setting. Separate infusion of bicarbonate is not a valuable option. Indeed, this could induce major side effects, as the concentration of such bicarbonate infusion is roughly 100-fold greater than the concentration in solutions. Combining a low sodium/ buffer-free and a normal sodium/bicarbonate buffered replacement solution optimizes metabolic equilibrium during citrate CVVH but at the cost of tight (i.e., every $4 \mathrm{~h}$ from onset) control. ${ }^{[1-4]}$

\section{HOW TO DETECT AND CORRECT CITRATE INTOXICATION AND OTHER SIDE EFFECTS OF CITRATE ADMINISTRATION?}

As mentioned earlier, the plasma citrate concentration depends on the balance between the rate of infusion and the loss by filtration and its metabolic degradation plus any losses through dialysis if CVVDF is used. Plasma citrate is mainly bound to calcium and, to a lesser extent, to other cations such as magnesium. Therefore, any accumulation 
of citrate may be associated with metabolic acidosis or metabolic alkalosis, depending on the metabolism and infusion rate. In case of metabolic acidosis, citrate may accumulate if liver and muscle fail to metabolize citric acid, a situation occurs in decompensated liver cirrhosis, severe shock, or after a prolonged cardiac arrest. Acid accumulates under these circumstances or-according to the Stewart approach-strong anions increase with subsequent metabolic acidosis. The classic anion gap probably rises in more than $95 \%$ of cases too, reflecting the increase in ionized citrate. However, lactate will mostly accumulate at the same time, which complicates this interpretation of events. With citrate binding to calcium, systemic ionized calcium concentration falls and the bound fraction of calcium rises. Total calcium remains constant or more often rises, depending on the amount of calcium infused to correct hypocalcemia. If calcium infusion is increased to correct low-ionized calcium levels, most of the calcium is captured by citrate. As a consequence, a disproportional rise in total calcium concentration occurs while ionized calcium remains low. Thus, the so-called calcium gap (total calcium minus ionized calcium) or the calcium ratio (total calcium/ionized calcium) always increases. A calcium gap of $>2 / 1$, in the absence of any obvious cause of metabolic alkalosis, very well indicates citrate accumulation. Once again, the use of a lower (e.g., $0.5 \%$ ) concentration of citrate should minimize the rate of metabolic complications. ${ }^{[1-6]}$ Therapy includes discontinuation of citrate infusions and replacement with normal sodium and/or bicarbonatebuffered substitution fluids. CVVH can generally be continued without anticoagulation and bicarbonatebuffered replacement, because patients with liver failure usually have coagulation disorders. In general, a 50\% dose reduction in liver failure should be sufficient to avoid early circuit clotting (a reduction of $50 \%$ of the citrate dose in a low-dose protocol will be enough in $99 \%$ of the cases and surely when using the predilution system as lower concentrations are used). Metabolic acidosis due to failure to metabolize citric acid can be diagnosed early by routine metabolic monitoring. It is corrected by discontinuing or tapering the dose of citrate and by adjusting the replacement fluid. In case of metabolic alkalosis, large amounts of citrate may enter the systemic circulation. If ultrafiltration flow gradually declines as a result of decreased filter performance induced by increased clogging, while blood flow is constant and also citrate infusion rate remains constant, less citrate and bicarbonate are lost by filtration and more citrate enters the systemic circulation. If liver function is normal, citrate is metabolized and metabolic alkalosis ensues as a result of an imbalance between loss and supply of citrate. Ionized calcium concentration may subsequently fall and the calcium ratio may rise proportionally because of alkalosis and not because of accumulation of citrate. A normal liver has a large reserve for citrate metabolism. Metabolic alkalosis at reduced ultrafiltration flow is better controlled by changing the filter instead of reducing the citrate dose, because this may cause filter clotting. Depending on blood and citrate flow, a definite lower limit of UF flow has to be protocolized, e.g., at a standard blood flow of $200 \mathrm{~mL} / \mathrm{min}$ and a citrate flow of $35 \mathrm{mmol} / \mathrm{h}$, the filter is changed when ultrafiltration flow remains lower than $2000 \mathrm{~mL} / \mathrm{h}$ or when the transmembrane pressure (TMP) is above $250 \mathrm{mmHg}$; at a blood flow of $150 \mathrm{~mL} / \mathrm{min}$ and a citrate dose of $18 \mathrm{mmol} / \mathrm{h}$, filter change is performed when ultrafiltration flow remains lower than $1500 \mathrm{~mL} / \mathrm{h}$ or when the TMP is above $250 \mathrm{~mm} \mathrm{Hg}$. The TMP threshold (above $250 \mathrm{~mm} \mathrm{Hg}$ ) is probably much better than the ultrafiltration flow as with the computerized system; it may not reflect a decline in filter porosity. As mortality in acute kidney injury (AKI) might improve at a higher renal replacement dose, continuing CVVH at lower UF flows is not advised. A TMP value must not be seen as "rough" and solitary number. In fact, the slope of the TMP curve will determine the membrane change, which implies that $250 \mathrm{~mm} \mathrm{Hg}$ is not a cut-off value per se. Monitoring of acid-base balance remains pivotal. Metabolic alkalosis resulting from accumulation of citrate because of a decline in ultrafiltrate flow at a fixed blood/ citrate flow relationship can be prevented by timely filter change. Nonetheless, accumulation of citrate with metabolic alkalosis and hypocalcemia may also occur if too much citrate is infused accidentally or if the patient has received a large volume of transfusion blood and UF flow is low. Hypocalcemia is observed when citrate accumulates and also when calcium is bound to citrate in the ultrafiltrate. During citrate in a CVVH mode, more calcium is lost in the ultrafiltrate than during CVVH running with heparin. In this case, hypocalcemia is not accompanied by a rising anion gap and calcium must be adjusted by infusion via a separate line. The required amount depends on the ultrafiltration flow and the calcium content of the replacement fluid. At a filtration flow of $2000 \mathrm{~mL} / \mathrm{h}$, a separate calcium infusion is not necessary if the replacement fluid contains calcium. However, this scenario is very rare and a calcium infusion through a central line must always be provided and adapted according to the ionized calcium blood levels. ${ }^{[1-6]}$

\section{PRE- OR POSTDILUTION AS THE IDEAL MODE? HOW SHOULD CITRATE BE GIVEN? SHOULD REPLACEMENT SOLUTION BE ADMINISTERED PRE, POST, OR DIVIDED BETWEEN PRE AND POST?}

In conventional hemofiltration, the replacement fluid bears resemblance to the electrolyte composition of plasma and extracellular fluid, except for a lower potassium content 
and a higher buffer content. In postdilution $\mathrm{CVVH}$, the trisodium citrate is administered before the filter and the commercially available replacement fluid contains either a low sodium/buffer-free solution, a normal sodium/ standard bicarbonate-buffered solution, or a combination of both at different dosages. ${ }^{[6-10]}$ The choice of the replacement fluid in postdilution CVVH depends on blood flow, UF flow, and patient's buffer needs and may change during the course of treatment. At a lower UF flow, less bicarbonate and citrate are filtered, resulting in increased uptake in the systemic circulation. At a UF flow of 2000 $\mathrm{mL} / \mathrm{h}$, the trisodium citrate does provide adequate buffer replacement so that a buffer-free replacement fluid is generally adequate. At a higher filtration flow, more citrate and bicarbonate are filtered. Thus, part of the replacement fluid should contain buffer. This obviates the need for a separate bicarbonate infusion, which should be discouraged because of side effects. In "older" citrate systems (with an high-dosed citrate infusion), using an high exchange flow of $4000 \mathrm{~mL} / \mathrm{h}$ could lead to acceptable metabolic control with a replacement solution with half buffer-free and half bicarbonate-buffered solution, although some cases require further refinement. Patients with severe acidosis and a high metabolic rate usually need full bicarbonate replacement from onset and the use of a high filtration flow. However, this is not true anymore in a "new" predilution citrate mode. Lower flow (with a cumulative pre- and postdilution of $35 \mathrm{~mL} / \mathrm{kg} / \mathrm{h}$ ) can indeed easily control acidosis even to the point of reducing bicarbonate in the postdilution fluid. The ratio between citrate and citric acid as well as the mode are probably key issues in this context. Guided by plasma acid-base state, replacement is then further adjusted to the needs. At higher citrate concentrations given separately, a flow in the range of more than $2000 \mathrm{~mL} / \mathrm{h}$ might be necessary to afford timely metabolic control and hemodynamic improvement. ${ }^{[1-6]}$

The above-described scenarios (adapting a flow of $4 \mathrm{~L}$ with "older" citrate solutions at higher concentrations) are based on a blood flow of $200 \mathrm{~mL} / \mathrm{min}$. This incurs filtration fractions above $25 \%$ when ultrafiltration rates are as high as $4000 \mathrm{~mL} / \mathrm{h}$. However, the lifespan of a good filter can be expected under higher filtration fraction rates when citrate is used. Citrate indeed has the capacity to dramatically reduce protein deposit and associated clogging processes at the inner side of the membrane. Changing blood flow at a constant citrate flow may lead to changes in sodium and citrate concentration in the filter and subsequently in the ultrafiltrate. Until recently, many commercially available fluids contained calcium. As the calcium in the replacement fluid counteracts the anticoagulant effect of citrate in the venous chamber, coagulation may be induced at this site. Therefore, the increasing popularity of introducing calciumfree replacement solutions is expected to benefit circuit survival time. Such solutions are already available in some European countries and await worldwide breakthrough. In the predilution, as opposed to postdilution, CVVH circuit initially described by Pallson (Table 1) ${ }^{[5]}$ citrate is added to a usually homemade calcium-free replacement fluid with normal sodium concentration. Calcium is supplied through a separate venous line. The solution described is formulated for a flow approximating $2000 \mathrm{~mL} / \mathrm{h}$. The system is simple except for the inconvenience that buffer and anticoagulant cannot be dosed separately, making it less flexible and intermingling dose and anticoagulation adaptation. In severely acidotic patients, the amount of buffer may be insufficient and a replacement solution containing both citrate and bicarbonate may be more appropriate. In that case, simplicity is lost. In the predilution option described by Hofmann, citrate is infused as a separate solution and a combination of normal and half normal saline is used as replacement fluid titrated in accordance with a patient's condition. Potassium is added to the replacement fluid, and calcium and magnesium are infused via a separate line. Additional supplementation of calcium is often necessary because more citrate-bound calcium is lost in the ultrafiltrate than during heparin-CVVH. The amount of supplementation depends on whether the replacement fluid contains calcium or not. Magnesium is probably lost as well. However, low concentrations of magnesium are seldom seen supposedly because of a compensatory shift from the intracellular compartment as has been observed in patients with severe burns. For supplementation, a standard calcium gluconate solution may be used and, eventually, a calcium-magnesium solution in case of magnesium deficit. It is not known which serum calcium concentration must be targeted because hypocalcemia is an inherent sign of critical illness and is possibly protective. The infused calcium might penetrate into the cells and enhance cytotoxicity. Aiming at an ionized Ca of $1.0 \mathrm{mmol} / \mathrm{L}$ (or between 4 \& $5 \mathrm{mg} / \mathrm{dL}$ ) is considered to be safe but still awaits proof by randomized studies. Values as low as $0.70 \mathrm{mmol} / \mathrm{L}$ (around $3 \mathrm{mg} / \mathrm{dL}$ ) are well tolerated but are not advocated in critically ill hemodynamically unstable patients. Citrate anticoagulation for CVVHDF (Table 3) is generally performed with calcium-free dialysate. However, Mitchell uses a calcium-containing dialysate as used in intermittent HD. As calcium will chelate part of the citrate in this condition, the citrate infusion rate is high relative to blood flow. Gabutti and Mitchel titrated the composition of the dialysate and/or replacement fluid to the arterial $\mathrm{pH}$. However, this implies that treatment dose has to be changed if $\mathrm{pH}$ changes (e.g., dialysate and filtration flow must be reduced at high $\mathrm{pH})$. In the predilution circuits with dialysis, citrate is generally infused separately and the composition of the replacement fluid is adjusted as good as possible. ${ }^{[10-16]}$ Yet, a predilution concentration of $0.5 \%$ 
$(18 \mathrm{~mol} / \mathrm{L})^{[17]}$ citrate can be used in a predilution volume of $1500 \mathrm{~mL}$ safely with a substitution fluid tapered to the body weight of a patient $(100-1200 \mathrm{~mL} / \mathrm{h}$ ) in order to achieve a cumulative dose of $35 \mathrm{~mL} / \mathrm{kg} / \mathrm{h}$. The protocol attempts a target dose of $35 \mathrm{~mL} / \mathrm{kg} / \mathrm{h}$ altogether (Figure 1). ${ }^{[17]}$

\section{IS THE STEWART APPROACH VALUABLE FOR MONITORING CITRATE ANTICOAGULATION AT THE BEDSIDE?}

Metabolic acidosis is a common disorder in patients with AKI on CRRT. The main source of $\mathrm{H}^{+}$in these patients comes from the metabolism of the sulfur-containing amino acids, methionine and cysteine. Oxidation of these compounds generates sulfuric acid, a strong acid (low pK) that dissociates and increases the levels of sulfate and $\mathrm{H}^{+}$. In the classic acid-base approach, bicarbonate is consumed by the generated $\mathrm{H}^{+}$. Therefore, the CRRT is seen as an alkalinizing therapy transferring bicarbonate from the replacement liquid into the blood to replenish the consumed bicarbonate. This results in an increase in $\mathrm{pH}$ and bicarbonate concentration and base excess. According to the Stewart-Fencl physicochemical approach, the measured strong ion difference (SIDm, i.e., the difference between measured strong cations and strong anions) controls the metabolic component of the acid-base status. As such, metabolic acidosis and alkalosis are reflected by, respectively, a decrease and increase in strong ion difference (SID). From this perspective, a patient who is placed on CRRT presents a decreased SID in pre-CRRT situation. CRRT acts to increase this reduced SID by enabling accumulation of other strong anions, such as sulfate and hippurate. Nevertheless, recent studies have shown that CRRT-induced alkalosis, at least to a certain level, is not harmful. As only independent variables can modify acidbase status in the Stewart-Fencl approach, CRRT would not cause bicarbonate flux from replacement liquid into blood but rather eliminate the accumulated strong anions. Serum chloride is normalized and SID restored, which translates into a secondary bicarbonate increase. Hence, bicarbonate stops being the main factor in the regeneration

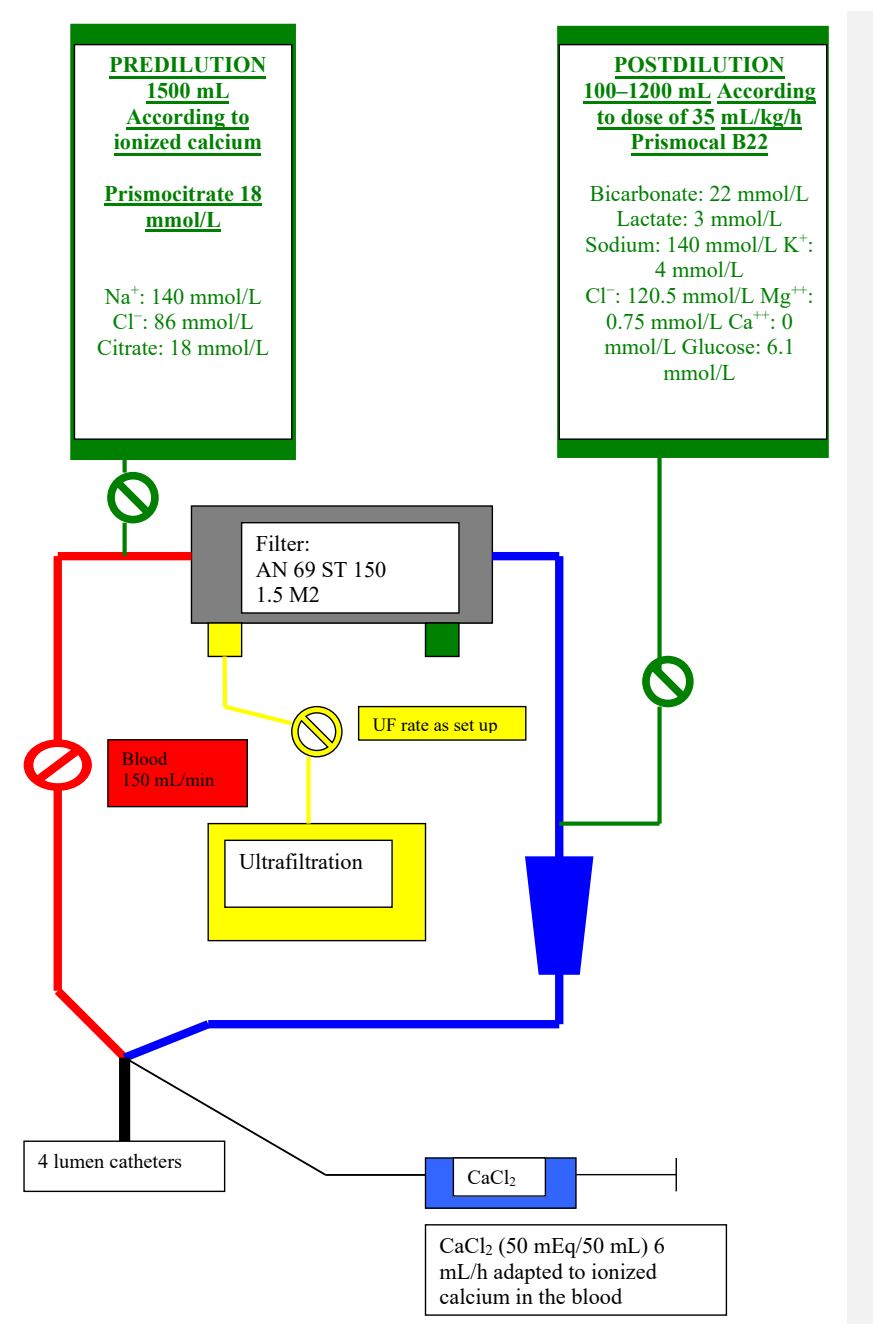

Figure 1: Anticoagulation regimen running with citrate in predilution as established in our unit. (Reprint by permission of Blood Purif 2015[17]) 
of acid-base status. In 1992, Fencl and colleagues developed a mathematical model to calculate SID and $\mathrm{pH}$ when the other independent variables $\left(\mathrm{pCO}_{2}\right.$, albumin, and phosphate) are known. This new definition of estimated strong ion difference (SIDe) is similar to the concept of Buffer Base (BB) developed by Singer and Hasting in 1948. The simplified equation, composed of $\mathrm{CO}_{2}$, anion charge of albumin, and anion charge of phosphate, is mostly used. Leblanc stressed the increment in SIDm in patients on CRRT or in patients with chronic renal failure during an intermittent HD session. On the other hand, other studies demonstrated a slight but significant decrease in SIDm (from 46.2 to $45 \mathrm{mEq} / \mathrm{L}$ ) during the HD session. This is in apparent contradiction to the expected increase in $\mathrm{pH}$ and bicarbonate concentration and base excess due to the alkalinizing effect of CRRT or HD. These findings were confirmed in patients followed for 1 year on daily "online" hemodiafiltration (HDF). How can this apparent contradiction between two variables that essentially measure the same effect be explained: because stable patients on periodic HD or CRRT usually have normal chloride levels, the unmeasured strong anions (sulfate, hippurate, etc.) are the ones that decrease. However, these anions are not taken into account in the measured SID formula, so that, apparently, this variable does not change. On the contrary, the SIDm will take into account the $\mathrm{CO}_{2}$ increase throughout the HD session or CRRT. Some studies found only a significant correlation between base excess and SID gap (or strong ion gap), but not between base excess and chloride and albumin and phosphates, and thus support the evidence that SID gap (or strong ion gap) is the only parameter associated with base excess regeneration. Other studies in critically ill ICU patients show similar results. However, when classifying patients in the high- and low-chloride groups, relating to this anion level in the dialysis fluid or the replacement solution, acidbase status correction (by means of variation of base excess) was significantly higher in the group with high chloride values. Furthermore, some studies claimed a correlation between base excess and SID gap (or strong ion gap) and chloride, while others emphasized the importance of chloride levels as an acidosis component in patients on HD or CRRT. This corroborates the hypothesis that correction of acid-base status during CRRT is not only due to the elimination of non-measured strong anions but also due to a normalization of serum chloride levels. Hyperchloremic acidosis can easily be confounded with sepsis-associated metabolic acidosis (e.g., when lactate is not available) but measuring SID will aid the clinician to make the difference. Measured SID, however, only allows to detect the increments in case of sodium increase without accompanying rise in chloride or a decrease in chloride. Such situations do not occur in typical ICU or chronic HDF patients. In HDF, the composition of the reinfusion liquid with an increased SID would accomplish the same objective: strong cations $\left(\mathrm{Na}^{+}\right)$with a smaller amount of strong accompanying anion (chloride) in an attempt to restore SID. Another important aspect is the albumin and phosphate anion charges included in the expression of total weak anions (ATot). ${ }^{[10-18]}$ This physicochemical

\begin{tabular}{|c|c|c|c|c|}
\hline Types & $\begin{array}{l}\text { Mean initial infusion of } \\
\text { citrate }(\mathrm{mmol} / \mathrm{h})\end{array}$ & Blood flow ( $\mathrm{mL} / \mathrm{min})$ & $\begin{array}{l}\text { Composition of restitution fluid } \\
\text { (mmol/L) }\end{array}$ & $\begin{array}{l}\text { Composition of } \\
\text { dialysate }(\mathrm{mmol} / \mathrm{L})\end{array}$ \\
\hline $\begin{array}{l}\text { CVVHDF } \\
\text { (Kutsogiannis, 2000) }\end{array}$ & 25 & 125 & $\begin{array}{l}\mathrm{Na}: 110 \\
\mathrm{Cl}: 110 \\
\mathrm{Mg}: 0.75 \\
\left(+\mathrm{HCO}_{3}: 16-50\right)\end{array}$ & $\begin{array}{l}\text { Na: } 110 \\
\mathrm{Cl}: 110 \\
\mathrm{Mg}: 0.75\end{array}$ \\
\hline $\begin{array}{l}\text { CVVHDF } \\
\text { (Gabutti, 2002) }\end{array}$ & 23 & 150 & $\begin{array}{l}\text { Citrate: } 13.3 \\
\mathrm{Na}: 139.9 \\
\mathrm{Cl}: 101.5 \\
\mathrm{Mg}: 0.75 \\
\text { (+ } \mathrm{K} \text { if needed) }\end{array}$ & $\begin{array}{l}\text { Citrate: } 13.3 \\
\mathrm{Na}: 139.9 \\
\mathrm{Cl}: 101.5 \\
\mathrm{Mg}: 0.75 \\
\text { (+ K if needed) }\end{array}$ \\
\hline $\begin{array}{l}\text { CVVHDF } \\
\text { (Dorval, 2003) }\end{array}$ & 25 & 125 & $\begin{array}{l}\text { Citrate: } 20 \\
\text { Na: } 145 \\
\text { Cl: } 100 \\
\text { Glucose: } 10 \\
\text { ( }+\mathrm{K} \text { if needed) } \\
\text { (+ } \text { if needed) }\end{array}$ & $\begin{array}{l}\mathrm{Na}: 154 \\
\mathrm{Cl}: 154\end{array}$ \\
\hline $\begin{array}{l}\text { CVVHDF } \\
\text { (Cointault, 2004) }\end{array}$ & 28.3 & 125 & $\begin{array}{l}\mathrm{Na}: 140 \\
\mathrm{Cl}: 109.5 \\
\mathrm{Mg}: 0.50 \\
\mathrm{Ca}: 1.75 \\
\text { Bicarbonate: } 32 \\
\text { Lactate: } 3\end{array}$ & $\begin{array}{l}\text { Na: } 140 \\
\text { Cl: } 109.5 \\
\text { Mg: } 0.50 \\
\text { Ca: } 1.75 \\
\text { Bicarbonate: } 32 \\
\text { Lactate: } 3\end{array}$ \\
\hline
\end{tabular}

CVVHDF: continuous venovenous hemodiafiltration. 
approach highlights the possibility that a decrease or an increase in these variables may cause metabolic alkalosis or acidosis, respectively. A number of studies showed that ATot as a whole was not modified throughout the session or the CRRT because the increment in albumin charge after the session was compensated by the decrease in the phosphate charge. The Stewart-Fencl approach allows to evaluate non-measured anions much more precisely than the anion gap. The latter, extensively used in the classic approach, presents remarkable interferences on the part of serum albumin and phosphate. As a consequence, correct evaluation of the type of metabolic acidosis becomes cumbersome, particularly in the presence of significant alterations in the ATot components, such as nephrotic syndrome, malnutrition, negative protein catabolism, and phosphorus intoxications and in ICU patients under citrate anticoagulation. In fact, the difference between SIDm and SIDe provides a quantity of nonmeasured anions (strong ion gap) free from the interference of weak acids. Studies by Kellum et al. on this strong ion gap revealed a poor correlation between anion gap and strong ion gap in critically ill ICU patients with low serum albumin concentrations, but this was not confirmed by other studies. ${ }^{[18]}$ It is highly probable that any correlation between anion gap and strong ion gap before and after HDF or CRRT is due to the normal albumin values throughout the session or the treatment. Therefore, the anion gap remains a valuable marker of non-measured anions in stable chronic HD patients and also in acutely treated CRRT patients, although less reliable in certain cases of AKI. Still, the strong ion gap is the parameter that best reflects non-measured anion accumulation during the interdialysis period or before CRRT. This parameter decreases significantly and, logically, includes anions from protein catabolism (mainly sulfate), which are adequately eliminated by HDF or CRRT. As opposed to anion gap, strong ion gap will not change either with $\mathrm{pH}$ or with changes in albumin and phosphate. In conclusion, it can be stated that the Stewart-Fencl approach does improve the evaluation of acid-base status in patients with AKI receiving citrate anticoagulation. In the presence of normochloremia, the measured SID does not reflect the alkalinizing effect of HD or CRRT. In these circumstances, the HD session or the CRRT treatment are perceived as non-metabolizable inorganic anion withdrawal (especially sulfate) techniques. The room left by these anions is replaced by $\mathrm{OH}^{-}$and secondarily by $\mathrm{HCO}_{3}^{-}$. The only advantage would be the evaluation of anions not measured by the strong ion gap, without the effects of albumin and phosphate. The fact that citrate can be considered as a weak acid (citric acid) or an unmeasured anion underscores the advantages of measuring the anion gap, the calcium gap, and the strong ion gap for adequate detection of its accumulation in ICU patients with citrate-treated AKI. The Stewart approach proves useful to detect a citrate intoxication in ICU patients with complex or mixed forms of acidosis and alkalosis. It stands to reason that when the direct measurement of citrate in the blood becomes routinely available, the detection of citrate accumulation will become much easier. ${ }^{[18]}$

\section{QUICK QUESTIONS AND ANSWERS: HOW TO CORRECT?}

\author{
How to detect and correct accumulation of citrate? \\ Origin: liver failure, poor muscle perfusion;
}

Metabolic Picture: metabolic acidosis, increasing anion gap, falling ionized calcium, rising calcium ratio (total calcium/ ionized calcium) or calcium gap (total calcium minus ionized calcium), increased lactate;

Correction: reduce or stop citrate, continue CVVH without anticoagulation or with low dose heparin, supply calcium (target ionized calcium should be $1.0 \mathrm{mmol} / \mathrm{L}$ or between 4 and $5 \mathrm{mg} / \mathrm{dL}$ ).

How to correct hypocalcemia?

Origin: accumulation of citrate.

Correction: correct cause mainly.

The cause can be increased loss of calcium, bound to citrate, by filtration.

Correction: infusion of calcium. As most critically ill patients have some degree of hypocalcemia, target ionized calcium should probably be subnormal, around $1 \mathrm{mmol} / \mathrm{L}$ or about $4 \mathrm{mg} / \mathrm{dL}$.

How to correct metabolic alkalosis?

Origin: too much bicarbonate substitution relative to filtration flow.

Correction: substitute more buffer-free and less bicarbonatebuffered solution.

Classical cause: declining filtration flow while citrate infusion is fixed or adjusted to blood flow

Treatment: change filter if filtration flow remains less than 1500-2000 mL (depending on blood and citrate flow).

Other causes: metabolic conversion from accumulated citrate if the liver recovers. 
Treatment: replacement with buffer-free solution or the citrate is down titrated to half (= halving the dose of citrate) especially in the predilution system.

If the cause is accidental or over-infusion of citrate: stop the citrate infusion, replace it with buffer-free solution, and increase filtration flow as necessary

How to correct hypomagnesemia?

Origin: increased loss of magnesium, bound to citrate, by ultrafiltration. Hypomagnesemia is generally not observed, probably because plasma magnesium is corrected by a shift from the intracellular compartment. In addition, the bicarbonate-buffered replacement fluid contains a high concentration of magnesium.

Correction: infusion of magnesium together with calcium.

Magnesium has to be maintained between 2 and $4 \mathrm{mmol} /$ dL.

How to correct accidental citrate intoxication?

Origin: if the cause is accidental with over-infusion of citrate or reduction of filtration flow below the lower limit at a constant citrate infusion rate.

Metabolic Picture: metabolic alkalosis, low ionized calcium, and proportional rise in $\mathrm{Ca}$ ratio as a result of alkalosis.

Correction: stop citrate.

Extremely high calcium ratios or gaps are described in the literature. In these cases, citrate hemofiltration was continued, while accumulation was evident. Low ionized calcium was treated with high doses of calcium infusion. This method is not advocated anymore.

Also, this type of intoxication is almost never seen with the predilution system and lower concentrations of citrate such as $0.5 \%$ or $18 \mathrm{mmol} / \mathrm{L}$.

Why and how to correct hypokalemia during CRRT with citrate?

Correction of hypokalemia with an infusion pump is very important as exposure of the body to combined hypokalemia, hypocalcemia, and hypomagnesemia may have dramatic effects. Therefore, potassium should be strictly monitored and maintained at levels between 4 and $5 \mathrm{mEq} / \mathrm{dL}$.

What to think about a steadily increasing infusion rate of calcium to the body?
Whenever, we do need to augment every $2-4 \mathrm{~h}$, the calcium infusion in the body - even when this is transient (or due to concomitant hypocalcemia and hypomagnesemia)

When going beyond $0.8 \mathrm{mg} / \mathrm{dL}$ in 4 to 6 hours, we have to think about other possibilities and surely than about possible citrate accumulation, although this might be a very slow process.

How to correct hypernatremia?

Origin: high citrate flow relative to filtration flow. Indeed, the flow gradually declines while citrate flow is constant. Citrate flow is high relative to blood flow. There is metabolic alkalosis as well.

Correction: change the filter if TMP is above $250 \mathrm{~mm}$ $\mathrm{Hg}$ (depending on the blood and citrate flow) and replace low-sodium, buffer-free solution.

Note: With the predilution system and low concentration of citrate, this is almost never encountered anymore.

How to correct hyponatremia?

\section{In old concentrated solutions}

Origin: substitution of solution with a too low sodium content. As this solution contains no buffer, metabolic acidosis may be present as well.

Correction: more substitution of normal saline, bicarbonatebuffered solution.

\section{In new less concentrated solutions}

Hardly seen anymore.

How to start the metabolic monitoring?

Minimal monitoring:

At 4-h interval: measurement of $\mathrm{Na}^{+}, \mathrm{K}^{+}, \mathrm{Cl}^{-}$, ionized calcium, blood gas analysis;

Calculation of anion gap and strong ion gap;

Once daily: total calcium for calculating the calcium ratio (total calcium/ionized calcium) or the so-called calcium gap (total calcium minus ionized calcium), magnesium;

Once or twice daily: creatinine, urea, phosphate.

When the patient becomes more stable, monitoring can be done at 6 -h intervals and eventually at 8 -h or even 12 -h intervals. 
Close monitoring is favored, as citrate doses may differ considerably between patients.

How to monitor anticoagulation in the circuit? (not necessarily daily but certainly at the start of treatment): post-filter ionized calcium (target $0.25-0.35 \mathrm{mmol} / \mathrm{L}$ or 0.8 to $1.3 \mathrm{mg} / \mathrm{dL}$ )

Again, monitoring is preferred, even if needed, less frequently.

\section{HOW TO CREATE A BEDSIDE CITRATE PROTOCOL THAT GUARANTEES PATIENT SAFETY?}

Predilution or postdilution: At present, predilution fluid with citrate is safer than postdilution fluid with bicarbonate.

Fluids: Commercial fluids should always be used.

Formulation of citrate. An equimolar infusion of citrate may have a different amount of sodium and buffer potential. These two issues must be considered separately.

Citrate infusion rate: A fixed rate adjusted to a fixed blood flow is chosen from the start. Thereafter, routine evaluation of anticoagulation in the circuit is necessary, particularly at the beginning. Adjustments are the rule in most cases.

Replacement fluid in postdilution. A buffer-free solution is generally adequate at filtration flows up to $2000 \mathrm{~mL} / \mathrm{h}$. A low bicarbonate (around $20 \mathrm{mmol} / \mathrm{L}$ ) buffered solution provides best metabolic control, especially at higher filtration flows and in severe acidosis, and surely, if you want to adapt the dose of $35 \mathrm{~mL} / \mathrm{kg} / \mathrm{h}$ to the body weight.

In the predilution system, a low concentration of bicarbonate of around $20 \mathrm{mmol} / \mathrm{L}$ must be targeted.

Calcium and magnesium infusion via a separate line; target ionized calcium is $1.0 \mathrm{mmol} / \mathrm{l}$ ( 4 to $5 \mathrm{mg} / \mathrm{dL}$ ). Infusion rate depends on filtration flow and concentration in the replacement fluids. Nowadays, calcium can be added to one of the solutions. Anyhow, a separate calcium infusion will be always mandatory.

To prevent metabolic alkalosis, change the filter if filtration flow drops below a set lower limit, for example, $1500 \mathrm{~mL} /$ min at a blood flow of $150 \mathrm{~mL} / \mathrm{min}$ or $2000 \mathrm{~mL} / \mathrm{min}$ at a blood flow of $200 \mathrm{~mL} / \mathrm{min}$ or when the TMP rises above $250 \mathrm{~mm} \mathrm{Hg}$. As said, TMP is the best tool for this monitoring. In the predilution system, the concentration of bicarbonate in the post-dilution fluid will be critical. As stated, a concentration around $20 \mathrm{mmol} / \mathrm{L}$ is optimal to avoid metabolic alkalosis.

Replacement fluid in predilution: A citrate-containing replacement solution may be sufficient in stable patients. A bicarbonate-buffered solution is most often required in patients with acidosis and at higher filtration flows. For practical reasons, one single solution must be chosen. In this context, a postdilution solution containing $20 \mathrm{mmol} / \mathrm{L}$ will be most satisfactory at least for the predilution system.

To prevent inadvertent filter clotting in predilution CVVH, the filter must be changed if the filtration flow drops to a set lower limit or whenever TMP rises above $250 \mathrm{mmHg}$. Although not recommended, an hemopermeability index may be used. Importantly, not the absolute value but rather the slope of the curve determines the efficacy.

In any case, nurses and physicians must be adequately trained. Most importantly, they have to learn to adjust the replacement fluid according to the measured changes in acid-base balance. The most important issue is to repeat and update training on a regular basis.

Adequate thrombosis prophylaxis is important.

New software including automatization may offer exact estimation of citrate loss and allows immediate adaptation of different variables through closed-loop systems.

Yet, a predilution concentration of $0.5 \%(18 \mathrm{~mol} / \mathrm{L})$ of citrate can be used at $1500 \mathrm{~mL}$ safely with a substitution fluid tapered to the body weight of the patient. The protocol seeks a target dose of $35 \mathrm{~mL} / \mathrm{kg} / \mathrm{h}$ (Figure 1).

\section{FUTURE SYSTEMS THAT MAY SIMPLIFY THE TASK OF CLINICIANS AND NURSING STAFF}

As said, today a new software, do give to the clinician, the possibility of automatization by knowing exactly the lost of citrate whenever modify one parameter and give the possibility to adapt immediately though the device computer nearly everything. This would give safety to a patient, flexibility to a physician, and less labor but more safety for a nurse. See comment above.

\section{CURRENT PRACTICE IN OUR ICU}

CRRT is performed under citrate anticoagulation in all patients. Patients are closely monitored (i.e., every $4 \mathrm{~h}$ at the beginning). Citrate dose is tapered in severe liver dysfunction without taking additional risks. A low concentration of citrate $(0.005-18 \mathrm{mmol} / \mathrm{L})$ is chosen 
in the predilution mode, starting at $1500 \mathrm{~mL} / \mathrm{h}$, which is adjusted afterwards according to the ionized calcium concentrations in the circuit. Postdilution fluid is enriched with $22 \mathrm{mmol} / \mathrm{L}$ bicarbonate to avoid rebound alkalosis. The amount of postdilution fluid is also tapered aiming always at a dose of $35 \mathrm{~mL} / \mathrm{kg} / \mathrm{h}$. Within the protocol, prewritten tables with adjusted doses are offered to nursing staff and attending physicians. A decisional tree is provided to guide therapy primarily in accordance to ionized calcium determinations in patient and circuit blood and also taking into account other variables such as blood gases, potassium, sodium, magnesium and phosphorus (Figure 1). ${ }^{[17]}$

\section{Conflict of Interest}

None declared.

\section{REFERENCES}

1. Oudemans-van Straaten HM, Ostermann M. Bench-to-bedside review: Citrate for continuous renal replacement therapy, from science to practice. Crit Care 2012;16:249.

2. Oudemans HM. Citrate anticoagulation. Blood Purif 2010;29:191-6.

3. Collin V. Citrate Anticoagulation. In: Extracorporeal circuits in reanimation. Les circulations extracorporelles en réanimation: SRLF-REANIMATION EUROPE. René Robert, Patrick Honore, Olivier Bastien, editors. General Editor: Elsevier Masson. Collection: Réanimation Europe. Amsterdam: Elsevier, 2006: 35-48.

4. Gabutti L, Marone C, Colucci G, Duchini F, Schönholzer C. Citrate anticoagulation in continuous venovenous hemodiafiltration: a metabolic challenge. Intensive Care Med 2002; 28: 1419-25.

5. Palsson R, Niles JL. Regional citrate anticoagulation in continuous venovenous hemofiltration in critically ill patients with a high risk of bleeding. Kidney Int 1999; 55: 1991-7.

6. Monchi M, Berghmans D, Ledoux D, Canivet JL, Dubois B, Damas P. Citrate vs. heparin for anticoagulation in continuous venovenous hemofiltration: a prospective randomized study. Intensive Care Med 2004; 30: 260-5.
7. Kutsogiannis DJ, Gibney N, Stollery D, Gao J. Regional citrate versus systemic heparin anticoagulation for continuous renal replacement in critically ill patients. Kidney Int 2005; 67: 2361-7.

8. Hyman A, Mendelssohn DC. Current Canadian approaches to dialysis for acute renal failure in the ICU. Am J Nephrol 2002; 22: 29-34.

9. Mehta RL, McDonald BR, Aguilar MM, Ward DM. Regional citrate anticoagulation for continuous arteriovenous hemodialysis in critically ill patients. Kidney Int 1990;38:976-81.

10. Tolwani AJ, Campbell RC, Schenk MB, Allon M, Warnock DG. Simplified citrate anticoagulation for continuous renal replacement therapy. Kidney Int 2001; 60: 370-4.

11. Asper R, Schwarzenhofer M, Derfler K, Zauner C, Ratheiser K, Kranz A . Impairment of citrate metabolism in acute hepatic failure. Wien Klin Wochenschr 1997; 109: 123-7.

12. Cointault O, Kamar N, Bories P, Lavayssiere L, Angles O, Rostaing L, et al. Regional citrate anticoagulation in continuous venovenous haemodiafiltration using commercial solutions. Nephrol Dial Transplant 2004; 19: 171-8.

13. Mitchell A, Daul AE, Beiderlinden M, Schäfers RF, Heeman U, Kribben $\mathrm{A}$, et al. A new system for regional citrate anticoagulation in continuous venovenous hemodialysis (CVVHD). Clin Nephrol 2003; 59: 106-14.

14. Dorval M, Madore F, Courteau S, Leblanc M. A novel citrate anticoagulation regimen for continuous venovenous hemodiafiltration. Intensive Care Med 2003; 29: 1186-9.

15. Jacobs R, Honore PM, Hendrickx I, Spapen HD. Regional Citrate Anticoagulation for Continuous Renal Replacement Therapy: All Citrates Are Not Created Equal! Blood Purif 2016;42:219-20.

16. Oudemans-van Straaten HM, Bosman RJ, Koopmans M, van der Voort $\mathrm{PH}$, Wester JP, van der Spoel JI, et al. Citrate anticoagulation for continuous venovenous hemofiltration. Crit Care Med 2009;37:545-52.

17. Jacobs R, Honoré PM, Bagshaw SM, Diltoer M, Spapen HD. Citrate Formulation Determines Filter Lifespan during Continuous VenoVenous Hemofiltration: A Prospective Cohort Study. Blood Purif 2015; 40: 194-202.

18. Kishen R, Honoré PM, Jacobs R, Joannes-Boyau O, De Waele E, De Regt $\mathrm{J}$, et al. Facing acid-base disorders in the third millennium-the Stewart approach revisited. Int J Nephrol Renovasc Dis 2014; 7: 209-17.

How to cite this article: Honore PM, De Bels D, Preseau T, Redant $\mathrm{S}$, Spapen HD. Citrate: How to get started and what, when, and how to monitor?. J Transl Intern Med 2018; 6: 115-27. 